\title{
Presentación Rara de Síndrome Antifosfolípido Primario Asociado a Hiperhomocisteinemia Como Causa de Enfermedad Cerebrovascular Isquémica Recurrente en Varón Joven
}

\author{
Rare Presentation Of Primary Antiphospholipid Syndrome Associated With \\ Hyperhomocysteinemia As A Cause Of Recurrent Ischemic Stroke In Young Male
}

\author{
Jesús Neyra-León, ${ }^{1,2}$ Juliana Aparcana-Machado ${ }^{3}$
}

\section{Resumen}

Se presenta el caso de un paciente varón de 38 años con antecedentes de enfermedad cerebrovascular isquémica recurrente sin etiología determinada y trombosis venosa en miembros inferiores. Debido a la diversidad etiológica del infarto cerebral en un adulto joven, se le hizo una serie de exámenes clínicos, con lo cual se obtuvo el diagnóstico de un síndrome antifosfolípido primario asociado a hiperhomocisteinemia.

Orientado el diagnóstico, se le dio terapia con anticoagulantes y pulsos de corticoides; posterior a lo cual mejoró. El Síndrome Antifosfolípido forma parte del diagnóstico diferencial en mujeres jóvenes con infarto cerebral, de las cuales la mayoría se ha reportado casos en su forma secundaria, pero encontrarlo en forma primaria y más aún en un paciente varón es raro. Asimismo, el aumento de valores de homocisteína está relacionada con la gravedad del primer evento cerebrovascular, mas no con eventos recurrentes.

Palabras clave: Síndrome antifosfolípido, infarto cerebral, adulto joven, homocisteína.

\section{Summary}

We present the case of a 38-year-old male patient with a history of recurrent ischemic cerebrovascular disease without determined etiology and venous thrombosis in lower limbs. Due to the aetiological diversity of cerebral infarction in a young adult, he underwent a series of clinical tests, which resulted in the diagnosis of a primary antiphospholipid syndrome associated with hyperhomocysteinemia.

Once the diagnosis was made, he was given therapy with anticoagulants and corticoid pulses; with posterior improvement. Antiphospholipid syndrome is part of the differential diagnosis in young women with cerebral infarction; most cases have been reported in its secondary form, but finding it in primary form and in a male patient is rare. Also, increased homocysteine values are related to the severity of the first cerebrovascular event, but not to recurrent events.

Keywords: Antiphospholipid Syndrome, cerebral Infarction, young adult, homocysteine. (source: MeSH NLM).

\section{Introducción}

El síndrome antifosfolípido (SAF) es una enfermedad sistémica autoinmune, cuyo principal proceso patológico es trombosis vascular tanto venosa como arterial junto con presencia de autoanticuerpos antifosfolipídicos. ${ }^{1}$ Este afecta más a mujeres $(80 \%)$ y aunque puede aparecer a cualquier edad, es más habitual entre los 20 a 40 años de edad. ${ }^{2}$ Se describe por lo general secundario a lupus eritematoso sistémico en un $30-50 \%$ de casos, pero puede aparecer de forma aislada como forma primaria, siendo las manifestaciones neurológicas como forma de inicio de la enfermedad no tan frecuentes en ambos casos.

Dentro de los criterios serológicos se requiere presencia de anticoagulante lúpico (LAC), anticardiolipinas (ACL) IgG o IgM (títulos mayores a 40 GPL o MPL) o anti ß2-glicoproteína-I (ß2-GPI) IgG o IgM positivos (+) (títulos mayores a percentil 99), en al menos 2 oportunidades, separadas por 12 semanas para el diagnóstico.,4

\footnotetext{
${ }^{1}$ Facultad de Medicina, Universidad Privada San Juan Bautista. Ica, Perú. ${ }^{2}$ Asociación de Investigación y Ciencia de los Estudiantes de Medicina de la UPSJB ICA. Ica, Perú

${ }^{3}$ Servicio de Neurología, Hospital Regional de Ica. Ica, Perú.
}

Correspondencia:

Jesús Andreé Neyra León

Dirección: Urbanización San Joaquín Nuevo X - 135, Ica - Perú.

Teléfono: (+51) 951836872

E-mail: jesus.24.47.1996@gmail.com 
El tratamiento se basa en fármacos anticoagulantes que son el único método para evitar una evolución recidivante que comprometa el pronóstico. Es importante tener la sospecha clínica para su diagnóstico y tratamiento, antes de un daño irreversible.,4

Presentamos el caso de un paciente varón de 38 años que presentó infarto cerebral recurrente como forma de presentación de un SAF primario asociado a hiperhomocisteinemia.

\section{Descripción del caso clínico}

Paciente varón de 38 años con un tiempo de enfermedad de aproximadamente 7 años de manera progresiva con infartos cerebrales isquémicos recurrentes y trombosis venosa profunda en miembro inferior derecho con tratamiento con Warfarina el cuál fue suspendido, asociado en el último año trastorno de comportamiento agresivo. Tiene como antecedentes familiares una hermana con lupus eritematoso sistémico (LES) y su padre fallecido a edad temprana por infartos cerebrales recurrentes.

A su ingreso al hospital presentó hemiparesia derecha, reflejo palmomentoniano presente, apraxia ideomotora, bradicinesia, incontinencia urinaria y momentos de disartria. Se le aplicó la escala de la evaluación cognitiva de Montreal (MoCA), el cual dio 20 puntos. También se encontró una solución de continuidad con presencia de lesiones costrosas y coloración oscura en el tercio inferior de pierna derecha (figura 1).

Se le practicó una ecografía doppler venosa de miembros inferiores donde se vio signos de trombosis subaguda a nivel de cayado safena femoral y a nivel de sistema venoso profundo derecho, así como una tomografía axial computarizada cerebral donde se evidenciaron áreas de hipodensidad difusas que comprometen ambos hemisferios cerebrales correspondientes a signos de isquemia crónica.

Durante su estancia hospitalaria cursa con comportamiento pueril, labilidad emocional, risas inmotivadas y actos impulsos motivo por el cual fue evaluado por psiquiatría dejando tratamiento antipsicótico.

En la resonancia magnética cerebral se evidenciaron lesiones compatibles con áreas isquémicas recientes en corteza cerebral y hemisferios cerebelosos (Figuras 2 y 3 ).

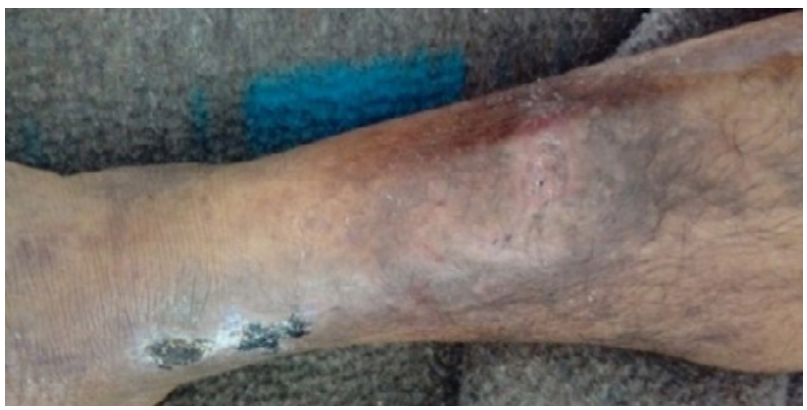

Figura 2. Solución de continuidad, con presencia de lesiones costrosas, rodeadas de áreas con coloración oscura en cara interna de tercio inferior de pierna derecha.
Se tomaron exámenes buscando el agente etiológico de los infartos cerebrales recurrentes dando los siguientes resultados: ANA negativo, Anti DNA negativo, Anti RO negativo, Antitrombina III: $102 \%$, Factor VIII: $70 \%$, Factor Von Willebrand: 158\%, Factor V Leiden Negativo, Anticoagulante Lúpico: Negativo, Homocisteína: $34.1 \mathrm{U} / \mathrm{ml}(\mathrm{VN}$ : 5-18 U/ml), Anticuerpos Anticardiolipina IgM: Negativo,
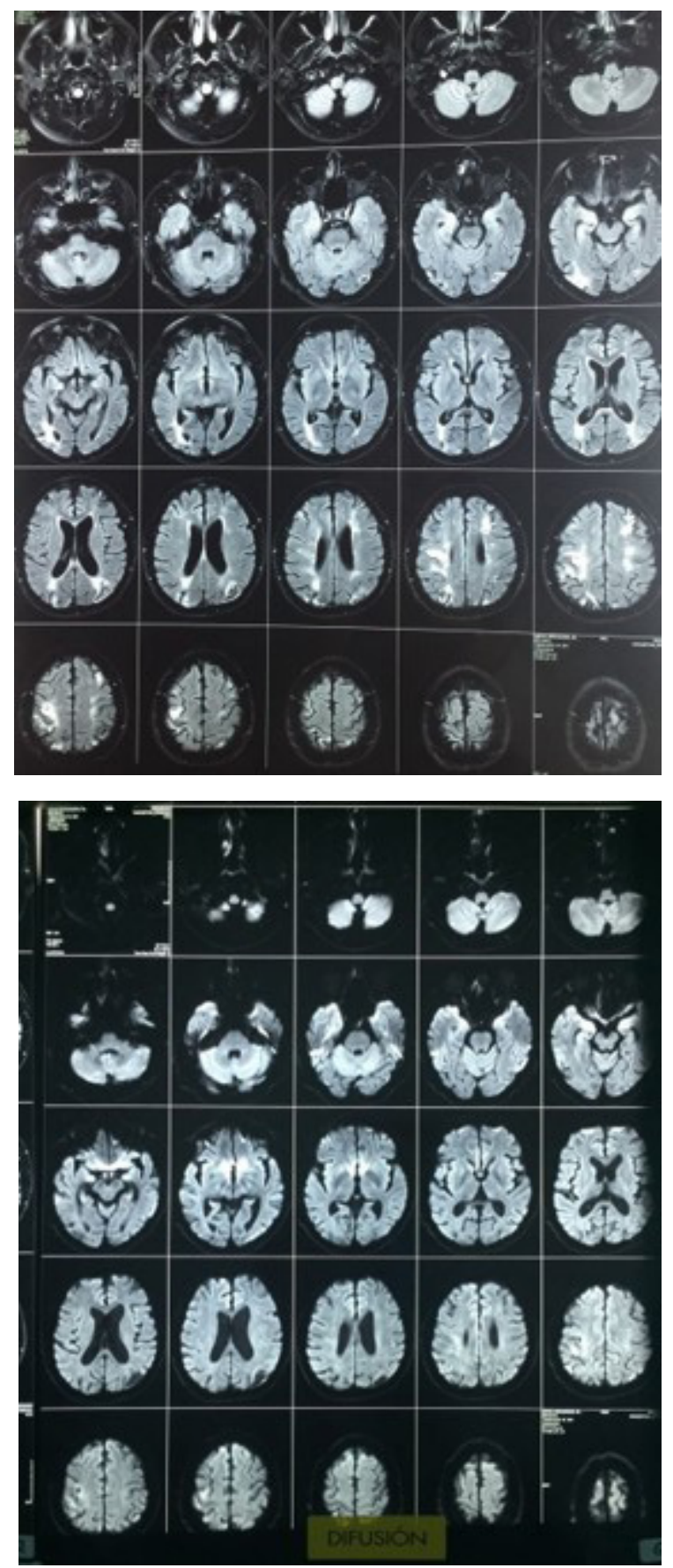

Figuras 2 y 3. En la resonancia en protocolo Flair y Difusión se evidencio lesiones difusas de la señal en la sustancia blanca subcortical y profunda de ambos hemisferios cerebrales con disminución del cuerpo calloso, las cuales presentan restricción leve a la difusión asimismo algunas presentan captación de la sustancia de contraste compatibles con áreas isquémicas. 
Anticuerpos Anticardiolipina IgG: Negativo y Beta 2 Glicoproteína 1, AC. IgG: $96.5 \mathrm{U} / \mathrm{ml}$ (Positivo > $5 \mathrm{U} / \mathrm{ml}$ ).

Se conversó con familiares del paciente quienes contaban con examen anterior de Beta 2 Glicoproteína 1, AC. IgG: $60.2 \mathrm{U} / \mathrm{ml}$ (Positivo > $5 \mathrm{U} / \mathrm{ml}$ ).

Se diagnosticó un SAF primario asociado a Hiperhomocisteinemia cuya forma de presentación de enfermedad son infartos cerebrales isquémicos recurrentes, con compromiso renal y vascular, se reinicia terapia anticoagulante (Warfarina $5 \mathrm{mg} /$ día) y pulsos de corticoides (Metilprednisolona $1 \mathrm{~g}$ /día durante tres días). Se citó después de un mes al paciente, evidenciándose mejoría.

Contamos con el consentimiento informado de los familiares del paciente para la realización de este reporte de caso.

\section{Comentario final}

Los casos de infartos cerebrales en adultos jóvenes considerados menores de 45 años, ocurren en alrededor de 3.4 a 11. 3/100.000 personas por año. Se describen diversas etiologías de esta condición; dentro de las que tenemos vasculitis, coagulopatías, enfermedad cardioembólica, entre otras causas. ${ }^{5,6}$

El SAF afecta más a mujeres $(80 \%)$ y puede aparecer a cualquier edad, pero es más habitual entre los 20 a 40 años de edad. ${ }^{1,2}$ Es una enfermedad que presenta diversas manifestaciones clínicas como trombosis venosas y arteriales; en la mayoría de los casos se encuentra asociado a una enfermedad autoinmune, usualmente lupus eritematoso, pero cuando no está asociado a esta patología o a otra condición se le conoce como SAF primario?

En nuestro caso vemos que se cumplió con criterios para un SAF primario como infarto cerebral isquémico recurrente, trombosis venosa profunda, marcadores lúpicos negativos y un marcador serológico como Beta 2 Glicoproteína 1, AC. IgG, el cual fue positivo en dos ocasiones separados por un intervalo mayor de 12 semanas. ${ }^{4}$

Los infartos cerebrales son una de las principales manifestaciones neurológicas en el síndrome antifosfolipídico, que cuando se vuelve recurrente lleva a daño neurológico diverso que se manifiesta clínicamente como trastornos cognitivos, manifestaciones neuropsiquiátricas, compromiso motor, entre otros, dejando secuelas irreversibles que limitan la calidad de vida. ${ }^{8}$ La anticoagulación oral a largo plazo es el tratamiento recomendado para prevenir recurrencias en estos pacientes, debido a que es eficaz y seguro. ${ }^{4}$

Como se había mencionado, los casos de SAF secundario a Lupus u otras enfermedades son muy reportados; sin embargo, las formas primarias de esta enfermedad no son muy frecuentes, ahí deriva la importancia de este caso. ${ }^{9}$ Por otro lado, la relación de la hiperhomocisteinemia encontrada en el paciente con los eventos cerebrovasculares isquémicos se ha visto en estudios donde niveles elevados de este aminoácido no presentan relación con episodios recurrentes, pero si influye mucho en la gravedad del primer evento cerebrovascular en el paciente. ${ }^{10,11}$

Concluimos que, ante pacientes adultos jóvenes con esta clínica, debe considerarse el síndrome antifosfolípido como posibilidad diagnóstica y así dar manejo precoz y evitar complicaciones.

\section{Referencias}

1. Sammaritano LR. Antiphospholipid syndrome. Best Pract Res Clin Rheumatol. 2019 Dec 19:101463.

2. Cervera R. Antiphospholipid syndrome. Thromb Res. 2017 Mar;151 Suppl1:S43-S47.

3. Liu Y, Zheng D, He Q. Heart, Brain, and Kidney Affected by Antiphospholipid Syndrome. J Clin Rheumatol. 2019 Dec 24.

4. Garcia D, Erkan D. Diagnosis and Management of the Antiphospholipid Syndrome. N Engl J Med. 2018 May 24;378(21):2010-2021.

5. Schneider S, Vibo R, Taba N, et al. Mortality in young adult patients with acute ischaemic stroke. Acta Neurol Scand. 2019 Dec 30.

6. Calvet D. Ischemic stroke in the young adult. Rev Med Interne. 2016 Jan;37(1):19-24.

7. Zhao JL, Sun YD, Zhang Y, et al. The clinical manifestations and thrombotic risk factors in primary antiphospholipid síndrome. Zhonghua Nei $\mathrm{Ke} \mathrm{Za}$ Zhi. 20161 de mayo; 55 (5): 386-91. doi: 10.3760 / cma.j.issn.0578-1426.2016.05.011.

8. De Amorim LC, Maia FM, Rodrigues CE. Stroke in systemic lupus erythematosus and antiphospholipid syndrome: risk factors, clinical manifestations, neuroimaging, and treatment. Lupus. 2017 Apr;26(5):529-536.

9. De Maeseneire Ch, Duray MC, Rutgers MP, et al. Neurological presentations of the antiphospholipid syndrome: three illustrative cases. Acta Neurol Belg. 2014 Jun;114(2):117-23. doi: 10.1007/s13760-0130275-6. Epub 2014 Jan 25.

10. Lehotský J, Tothová B, Kovalská M, et al. Role of Homocysteine in the Ischemic Stroke and Development of Ischemic Tolerance. Front Neurosci. 2016 Nov 23; 10:538. eCollection 2016.

11. Kumral E, Saruhan G, Aktert D, et al. Association of Hyperhomocysteinemia with Stroke Recurrence after Initial Stroke. Stroke Cerebrovasc Dis. 2016 Aug;25(8):2047-54.

Contribuciones de los autores: JNL y JAM participaron en la concepción, diseño, redacción, revisión crítica y aprobación de la versión final del artículo

Fuente de financiamiento: Los autores declaran no haber recibido ninguna financiación para la realización de este trabajo.

Conflictos de interés: Los autores declaran no tener conflicto de interés con la publicación de este artículo. 